\title{
GEODATOS Y PAISAJE: DE LA NUBE AL AULA UNIVERSITARIA ${ }^{1}$
}

\author{
María Luisa de Lázaro y Torres \\ Departamento de Geografía Humana. Universidad Complutense de Madrid \\ mllazaro@ucm.es \\ Sara Izquierdo Álvarez \\ Instituto CEU de Estudios Históricos \\ sizquierdo@uch.ceu.es \\ María Jesús González González \\ Departamento de Geografía y Geología. Universidad de León \\ mjgong@unileon.es
}

\section{RESUMEN}

La Geografía es una ciencia que integra con facilidad las últimas novedades tecnológicas en el ámbito de la investigación, en la docencia y en la práctica profesional. Novedosas herramientas de visualización existentes en la Nube facilitan nuevos resultados de investigación y favorecen el aprendizaje y una mejor comprensión del paisaje y sus tipologías. Este se muestra mediante la técnica del storytelling aplicada a itinerarios geográficos y a la profundización sobre los paisajes agrarios.

Palabras clave: geodatos, visualización cartográfica, geolocalización, geotecnologías, ArcGIS Online, storytelling, España, Madrid, paisaje.

\section{ABSTRACT}

Geography is a science that easily integrates the latest technological developments, both in the field of research and in teaching and professional practice. Innovative visualisation

Fecha de recepción: marzo 2014.

Fecha de aceptación: diciembre 2014.

1 Este artículo se enmarca en el trabajo relacionado con el proyecto europeo: School on Cloud: connecting education to the Cloud for digital citizenship (543221-LLP-1-2013-1-GR-KA3-KA3NW) (2013-2016) y con el Proyecto de Innovación y Mejora de la Calidad Docente financiado por el Vicerrectorado de Evaluación de la Calidad de la UCM «Aprender Geografía con la Web 2.0 a través de la evolución de los paisajes agrarios de España» (PIMCD 98/2014). 
tools available on the cloud facilitate new research results, and also promote learning and a better understanding of the landscape and its typologies. This is demonstrated by employing the technique of storytelling, geographical itineraries and agrarian landscapes.

Keywords: geodata, cartographic visualisation, geolocation, geotechnologies, ArcGIS Online, storytelling, Spain, Madrid, landscape.

\section{INTRODUCCIÓN}

La mayoría de los datos existentes en la actualidad tienen una componente geográfica, de ahí que se hable frecuentemente de geodatos. Un geodato es un dato que contiene una referencia espacial y, por tanto, se puede localizar en un mapa o en un plano. Puede además contener metadatos que son datos que describen otros datos. Existen protocolos o acuerdos, como por ejemplo en la Web 3.0 o Web semántica, sobre cómo crear los metadatos y en qué orden se deben introducir los datos que serán parte de los metadatos, con la finalidad de facilitar las búsquedas de contenidos, sin que la herramienta o la lengua empleada sea una dificultad.

Es posible visualizar los datos en un mapa (cartografía) o en un gráfico (estadística) y que ambos se integren empleando los mapas digitales, lo que ofrece la posibilidad de navegar por los datos y los mapas. Ello ha supuesto no sólo un avance fundamental en las Tecnologías de la Información Geográfica o geotecnologías, sino un reto para la enseñanza y el aprendizaje de la Geografía en las aulas universitarias. Este uso de los datos derivado de los avances de las técnicas cartográficas y estadísticas constituye el elemento esencial de una nueva praxis científica y de un cambio en la Geografía que tiene rango epistemológico (Moreno, 2013), y como tal deberá afectar al proceso de enseñanza-aprendizaje de la Geografía y a las metodologías docentes empleadas en las aulas. Además, permite hallar nuevas conclusiones enriqueciendo y matizando los resultados de la investigación. Algunos autores hablan de la revolución de la información geográfica (IG) o geoinformación (González y Lázaro, 2011) de la que la docencia también se enriquece (De Miguel, 2013 y 2014).

Cada vez es posible manejar un mayor volumen de datos, lo que nos hace mencionar algunos términos de uso generalizado en el ámbito anglosajón: big data (grandes conjuntos de datos), data mining (minería de datos) o, si es la propia población la que produce los datos, crowdsourcing data entry (los ciudadanos aportan datos de forma masiva). Todo ello es posible gracias a la mejora incesante de los ordenadores y de los programas; al crecimiento y evolución de Internet (Web 2.0, Web 3.0, Web 4.0...), que ofrece un número cada vez mayor de herramientas que permiten la participación ciudadana, el intercambio de datos y el acceso a servicios, pasándose a emplear el término Cloud Computing o computación en la Nube; y a la implementación del acceso a la información geográfica.

La creciente disponibilidad de datos en general y de geodatos en particular, a través de la Nube de Internet, ha incrementado su utilización, y también la demanda de los mismos, lo que ha favorecido la importancia de las tecnologías espaciales y el aumento del número de puestos de trabajo relacionados con el sector. Algunos programas internacionales impulsan todo ello, como son la Agenda Digital 2020 de la UE, que pretende fomentar este campo 
científico-tecnológico; la reciente iniciativa de la ONU Global Geospatial Information Management, UN-GGIM y la Directiva INSPIRE ${ }^{2}$, entre otros.

El movimiento open data, en relación a la información espacial, que está impulsado por las Infraestructuras de Datos Espaciales (IDE) y la Directiva INSPIRE, ofrece nuevas posibilidades a la investigación y a la docencia. En ese marco el acceso a la información geográfica se realiza de diversas formas, entre las que podemos señalar:

1. Descargas directas, que desde distintos servidores en Internet permiten obtener ficheros que contienen capas de información geográfica.

2. Conexión a estos mismos servidores mediante servicios del Open Geospatial Consortium $(O G C)$ a través de un programa específico para ello. Según el servicio seleccionado podemos visualizar las capas de información o bajarlas desde la Nube de Internet a nuestro terminal.

3. Obtención de información empleando los Sistemas Globales de Navegación por Satélite (GNSS) como el GPS americano o el Glonass ruso principalmente, frecuentemente disponibles en aplicaciones ofrecidas por nuestros propios dispositivos móviles (teléfonos inteligentes, iPads, tabletas...)

Estas emergentes tecnologías, sólo posibles por la existencia de la Nube de Internet, suponen un avance de sistemas inteligentes que, debidamente aprovechados, ofrecen grandes posibilidades en la mejora del pensamiento espacial y en la acción positiva sobre el territorio, por ejemplo a través de una retroalimentación de la ciudadanía a los datos ofrecidos por los organismos públicos. Si todo ello es aplicado a las ciudades, podríamos hablar de ciudades inteligentes o smart cities y si se aplica a la docencia podemos hablar de smart learning, aunque también podemos observar que las smart cities exigen nuevos aprendizajes, para los cuales el acceso a la información y a los geoservicios son elementos fundamentales.

Así, en este trabajo se muestra cómo el crecimiento de las herramientas de visualización cartográfica (geotecnologías) en la Nube aplicadas a los resultados de cualquier investigación que tenga un componente territorial relacionado con la localización, facilitará la obtención de nuevas conclusiones y mejorará el pensamiento espacial, en la línea de las reflexiones que sobre el mismo realizaron Lee y Bednarz (2009). Para ello se ha partido de los resultados de los Proyectos de Innovación y Mejora de la Calidad Docente de la UCM «Aprender Geografía con la Web 2.0» (PIMCD 133/2011), «Aprender Geografía de España con la Web 2.0» (PIMCD 165/2013)3 , siguiendo la línea de lo trabajado en proyectos anteriores y los resultados de su empleo en el aula universitaria. En ellos se ha empleado la técnica del storytelling, que como se explicará más ampliamente en el apartado III, supone enseñar

2 INSPIRE, Infraestructure for Spatial Information in Europe, es la directiva sobre la Infraestructura de Datos Espaciales de Europa, aprobada por el Parlamento Europeo y el Consejo el 14 de marzo de 2007 (Directiva 2007/2/CE)

3 Proyectos competitivos financiados por la UCM - Campus de Excelencia Internacional coordinados respectivamente por la Dra. D a María Luisa de Lázaro y Torres y por el Dr. D. Miguel Angel Alcolea Moratilla. En ellos participan profesores de distintas Universidades españolas (Complutense de Madrid, León, Málaga y Zaragoza) y de distintos departamentos dentro de la misma UCM (Departamento de Geografía e Historia, y Departamento de Análisis Geográfico Regional, de la Facultad de Geografía e Historia; y Departamento de Didáctica de las Ciencias Sociales (Geografía, Historia, Historia del Arte), Facultad de Educación-Centro de Formación del Profesorado). 
y aprender mediante una narración basada en la visualización de datos en forma de mapas, gráficos, vídeos, etc., en este caso, empleando la plataforma de ArcGIS OnlineTM, Esri®.

Comenzaremos mostrando las posibilidades que ofrecen algunas herramientas y plataformas que permiten localizar y visualizar paisajes de interés y mejorar su conocimiento mediante el trabajo con los geodatos. Nos centraremos en la plataforma de ArcGIS Online (AGOL). Después realizaremos una introducción a la citada técnica del storytelling y su adecuación para una aproximación a los paisajes en geografía. Todo ello se aplicará a dos formas de aproximación al paisaje, una primera basada en itinerarios geográficos y otra basada en el comentario de imágenes de paisajes relacionadas con aspectos agrarios localizadas en un mapa colaborativo. De este modo, se pretende abrir nuevos horizontes para la investigación y la docencia, aprovechando sinergias y una mejor integración del trabajo en la Nube de Internet en las aulas universitarias. Terminaremos con las conclusiones obtenidas derivadas del empleo generalizado de estas geotecnologías emergentes.

\section{HERRAMIENTAS Y PLATAFORMAS PARA LA VISUALIZACIÓN DE GEODATOS}

Cada vez existe una mayor variedad de herramientas de visualización de geodatos. Un número creciente de ellas son plataformas y servicios para publicar datos en la Nube. Algunas las podemos catalogar como SIGWeb, que integran las herramientas propias de los Sistemas de Información Geográfica (SIG) con el trabajo en la Nube (ArcGIS Online, Iberpix, globos virtuales...). La mayoría de ellas permiten además descargas de datos georreferenciados o geodatos, lo que nos permite considerar las SIGWeb como una cuarta forma de obtención de información geográfica a añadir a las señaladas anteriormente.

Podemos señalar, aportando algunos ejemplos concretos de su empleo, y sin pretender ser exhaustivos, otras tecnologías que permiten una visualización de datos: Stat Planet (Stat Silk), empleada para difundir los datos de 2012 del Índice de Desarrollo Humano (IDH); Geoclip, que se ha utilizado en el Atlas Nacional de Francia (géoportail); y Flex, con la que se realizó el visor de CartoCiudad, entre otras.

Un creciente número de tecnologías de código libre, como GeoNode o Geoserver, empleado en el Atlas de la Vivienda en España, están al alcance de cualquiera en Internet sin coste alguno. Además, podemos visualizar mapas con CartoDB, GeoCommons, GISCloud, Google Maps Engine Lite, Mango, MapBOX, Polymaps, QGIS Cloud, Worldmap, Infographs, Infogr.am, Ushahidi, TileMil, OpenHeatMap, Openlayers y DataViz...

Otras herramientas permiten visualizar los datos en gráficos e integrar esos gráficos y datos en la cartografía, y en muchos casos, compartirlos, como sucede con Google Public Data Explorer, Gap Minder, Many Eyes, Tableau Public, Gephi, etc.

La extendida tecnología ESRI nos ofrece la plataforma de ArcGIS Online (AGOL), que es la que vamos a emplear en este trabajo, por algunas de las ventajas que ofrece y que pasamos a exponer, después de una breve caracterización de la misma.

Se trata de una plataforma basada en la Nube de Internet, rápida y sencilla, que permite compartir el trabajo cooperativo en la «Nube» y en la «Tierra» (aula universitaria). Tiene dos modelos de usuarios derivados del tipo de suscripción que ostentan: uno, de carácter gratuito o de libre acceso para investigadores y docentes, y otro de pago para organizaciones o instituciones. En este segundo caso existen diferentes niveles de usuarios o figuras. El administrador 
de la plataforma para instituciones, que es el que da de alta a los usuarios y toma algunas decisiones, como por ejemplo las unidades por defecto que aparecerán en la cartografía que se elabore; el publicador, que puede realizar aplicaciones web y ofrecer servicios, y el usuario, que únicamente puede realizar cartografía. Permite crear una cartografía muy completa enriqueciendo los datos propios con capas de información de diversas procedencias, facilitando así la reutilización de información pública y un trabajo colaborativo sin precedentes. Para ello ESRI ofrece gratuitamente algunas plantillas que se dedican de forma específica a la técnica de cómo contar historias (storytelling maps), permiten comparar imágenes y/o mapas e integrar nuestros datos en un visor básico de amplias prestaciones. También se pueden enlazar distintas plantillas, por ejemplo la del perfil topográfico en el visor básico. Con ellas podemos compartir la información que queramos, donde y con quien queramos: en un blog, integrado en una página web preexistente, o creando una página web propia, por ejemplo, sin necesidad de hacer otro trabajo que el de la estricta elaboración de nuestros propios datos, para después añadirlos a otra información previamente disponible en la plataforma.

La interface o herramienta permite además integrar gráficos en los mapas (figura 1), a modo de ventana emergente que se activa con un simple clic del ratón. Sus prestaciones y su usabilidad o entorno de fácil manejo, amigable e intuitivo, mejoran cada día, permitiendo realizar mapas a partir de herramientas de Office con la aplicación ESRI Maps for Office, y estando integrada en ArcGIS Desktop a partir de la versión 10.1, lo que permite trabajar sobre la plataforma de AGOL y emplear las mismas capas de información desde distintos programas.

En este trabajo se presentarán los geodatos relacionados con los itinerarios y los relacionados con los paisajes agrarios, y serán visualizados en AGOL, a partir de la aplicación web creada con ellos. De esta forma la cartografía pasa de ser algo estático a integrarse en la Nube, con lo que es posible navegar e interactuar con ella y, además, ampliarla y actualizarla continuamente, incluso, si se programa para ello, por distintos usuarios.

Figura 1

GRÁFICOS INTEGRADOS EN LA CARTOGRAFÍA

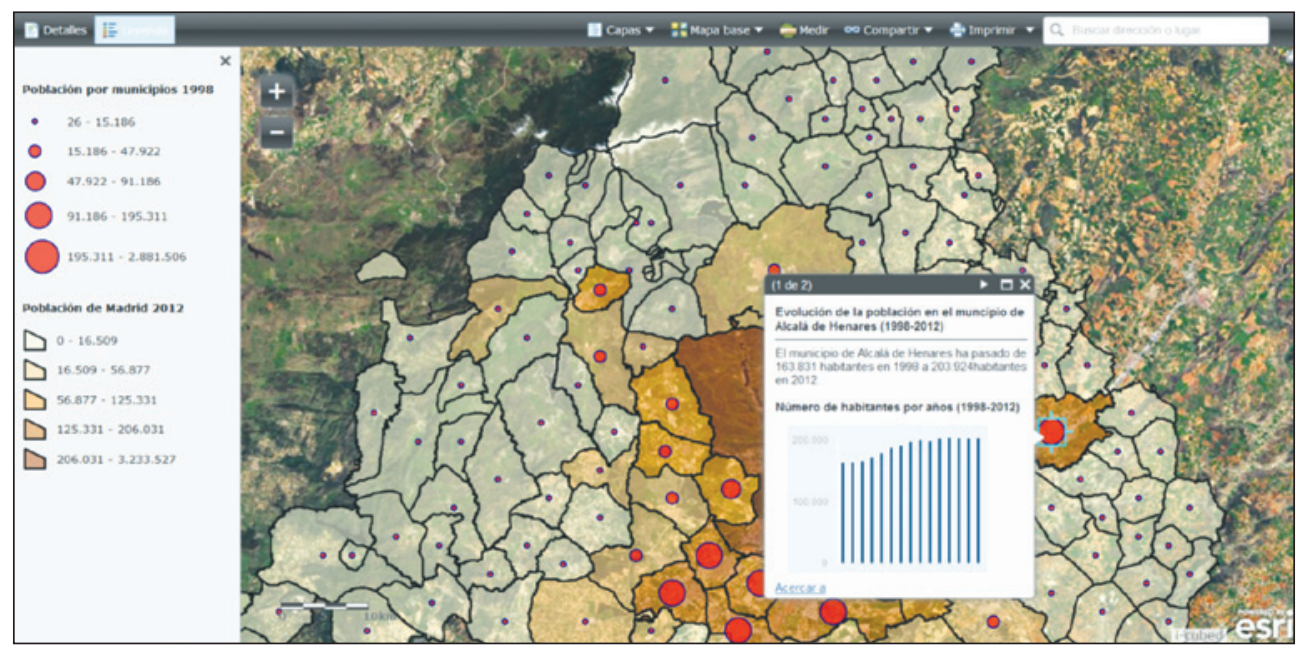

Fuente: Elaboración propia en ArcGIS Online a partir de los datos del INE. 


\section{LA TÉCNICA DEL STORYTELLING}

La revalorización de la importancia del paisaje (Zárate, 2011) desde la publicación del Convenio Europeo del Paisaje (Florencia, 2000) y la diversidad de paisajes en España han hecho que se aborden en distintos soportes y formatos, como por ejemplo en forma de atlas (Mata y Sanz, 2003; Campano, 2009; Pons, 2011), de observatorios (Junta Andalucía, 2007) o estableciendo tipologías de paisajes rurales (Molinero, Ojeda y Tort, 2011). Además, la aproximación al paisaje se puede realizar a través del arte con determinados hitos, como por ejemplo, los cuadros de un pintor (Espejo y López de los Mozos, 2012) o a través de determinados hechos literarios o históricos que pudieron acaecer. Si bien, la novedad del trabajo que aquí se presenta se basa en la opción de navegar online por el propio paisaje virtual, con la posibilidad de que cualquier miembro del equipo de trabajo pueda integrar, en cualquier momento, nueva información sobre el mismo. En todos los casos, la observación del paisaje (Liceras, 2003), como fuente de primera mano para su mejor conocimiento, resulta imprescindible.

En este trabajo se realiza una observación y visualización del paisaje que se plasma a través de los geodatos resultantes de investigaciones que emplean herramientas digitales. Estos geodatos serán los puntos de interés marcados en un itinerario que nos permitirán aproximarnos por un lado a los propios resultados de la investigación y por otro al paisaje evocado por ella. Esto se puede hacer a través de la técnica del storytelling, que es una metodología que elabora una narrativa con un hilo conductor preciso ayudándose de fotografías, vídeos, sonido... que nos ayuden a responder cuestiones como qué, dónde, cuándo y porqué (Ho, Åström y Jern, 2013).

Una experiencia cartográfica digital exige el manejo de conceptos y técnicas a través de una aplicación web para configurar los elementos fundamentales de un paisaje y los conceptos que entraña. Es una experiencia de comprensión del paisaje con oportunidades tecnológicas y conceptuales.

Según la National Storytelling Association, storytelling es el arte de usar el lenguaje, la manera de comunicar las ideas a otros, de tal manera que lo que se transmita consiga llamar la atención de nuestros receptores.

Las historias tienen el poder de llegar a nosotros y nos transportan a la atemporalidad. Son una manera de pensar, un organizador principal de la información y las ideas, el alma de una cultura, y la conciencia de un pueblo. Las historias nos incitan a saber, recordar y entender (Livo y Rietz, 1986), y al mismo tiempo, reflejan nuestras percepciones sobre esa realidad territorial del paisaje que se narra.

Se trata de una técnica antigua pero novedosa. Aunque la narración siempre ha estado presente en el ámbito educativo, hasta hace poco no era considerada una herramienta de aprendizaje-enseñanza, más bien se utilizaba para expresar opiniones, compartir experiencias, entretener y conectarse con otros. Cuando éstas se utilizan con un fin educativo, en nuestro caso para analizar el paisaje, podemos fomentar diversidad de aspectos como: el trabajo cooperativo, relacionar la teoría con la práctica, el pensamiento crítico, la construcción de nuevos conocimientos, revelar múltiples perspectivas... (Alterio, 2002).

La narración conocida como storytelling está experimentando actualmente un renacimiento considerable. Esto ha llevado a muchos educadores a pensar en las formas en que la narración se puede utilizar para explorar temas y visiones importantes compartidas. La actual 
preocupación por las cuestiones medioambientales se conecta con este renacimiento, ya que los cuentos populares acerca de la relación entre la Tierra y sus habitantes humanos han estado en el centro de la narración desde los tiempos más remotos. No sólo estas historias ofrecen una fuente de inspiración, también contienen un alto potencial para la comprensión de las muchas maneras en que valoramos y devaluamos nuestro hermoso planeta verde y azul. Las historias nos proporcionan una visión práctica de los diferentes enfoques de nuestras dificultades ambientales más persistentes (Beddes y Johnson, 1988).

Para la creación de historias se requiere un proceso muy cuidadoso. No vale solo el decir: tenemos esta historia, por tanto la relatamos. Todo tiene que pasar por un transcurso de tiempo y de reflexiones. Debemos pensar si se va a analizar mejor el paisaje, si el grupo de estudiantes al que va a ir dirigido aprenderá mejor con esa técnica, qué forma de contar historias se adapta mejor a esa clase, qué resultados pretendemos lograr, cómo los vamos a valorar y si necesitamos más medios (Alterio, 2002). Así, fomentamos habilidades de pensamiento crítico, vocabulario y patrones de lenguaje que se van a ir mejorando mediante el uso de historias (Zabel, 1991).

Las investigaciones sugieren claramente que los maestros deben estimular y enriquecer el desarrollo verbal en los colegios. En las universidades se sigue defendiendo una buena clase magistral en el momento oportuno. Egan (1993) afirmaba que la expresión oral y la escrita no son opuestas, sino que el desarrollo de esa expresión oral es la base necesaria para el posterior desarrollo de la alfabetización.

Hay una gran diversidad de historias digitales, pero las podemos agrupar en tres grandes grupos: narrativas personales, que son narraciones sobre acontecimientos de incidentes significativos en nuestra vida; documentales históricos, que versan sobre eventos que nos ayudan a entender el pasado; e historias diseñadas para informar e instruir sobre una visión particular de un concepto o práctica (Robin, 2006).

Tenemos cuatro formas de contar las historias, la visual, la oral, la escrita y la física. Podemos utilizar la visual para confeccionar un documental histórico- paisajístico, dentro de la narración digital (digital storytelling), incluyendo audio, video, imágenes, texto...

A pesar del énfasis en la tecnología informática, la narración digital no es una práctica nueva. Uno de los pioneros más destacados del campo es Joe Lambert (2013) el co-fundador del Center for Digital Storytelling (CDS), una organización no lucrativa, formada por la comunidad artística en Berkeley, California. El CDS ha estado ayudando a los jóvenes y adultos en la creación y el intercambio de relatos personales a través de la combinación de la escritura reflexiva y herramientas de medios digitales desde la década de 1990 (Robin, 2006).

La investigación ha demostrado que el uso de multimedia, el juego y el storytelling (Eck, R. 2006) en la enseñanza ayuda a los estudiantes a retener nueva información, y a comprender mejor el material difícil. La narración digital puede proporcionar a los educadores una potente herramienta para mejorar el aprendizaje de sus alumnos.

El Center for Digital Storytelling (CDS) pone de manifiesto los siete elementos fundamentales en la narración para que tenga éxito:

1. Punto de vista. ¿Cuál es el punto principal de la historia y cuál es la perspectiva del autor?

2. Una pregunta dramática. Una pregunta clave que mantiene la atención del espectador y será respondida al final de la historia. 
3. Contenido emocional. Problemas graves que cobran vida de una manera personal y poderosa y se conecta a la audiencia con la historia.

4. El don de voz. Una forma de personalizar la historia para ayudar al público a entender el contexto.

5. El poder de la banda sonora de música u otros sonidos que apoyan y embellecen la historia.

6. El contenido suficiente para contar la historia sin sobrecargar al espectador.

7. Ritmo. El ritmo de la historia y la forma en que progresa (lenta o rápidamente).

Jill Eck (2006) concluye que el storytelling es una herramienta de aprendizaje eficaz y un gran método de enseñanza. Se observó cómo la utilización de la narración provocaba en los alumnos un interés por el proceso de aprendizaje y un desarrollo del aprendizaje reflexivo. Asimismo nos revela que el alumnado fue capaz de conservar y recuperar la memoria una semana después de la primera lección. Pero lograr este éxito va a depender de las habilidades narrativas del profesor y de la relevancia de las historias y nos podemos ayudar situando estas historias en un mapa, para lo que vamos a utilizar en el trabajo que se presenta la plataforma AGOL. Esta herramienta la vamos a emplear para presentar un paisaje a través de un itinerario, y para profundizar sobre los paisajes agrarios.

\section{ELABORACIÓN DE ITINERARIOS A TRAVÉS DE GEODATOS SOBRE DIVERSOS PAISAJES ESPAÑOLES}

En el primer caso que se presenta se emplea, como punto de partida, el itinerario geográfico. La aproximación al paisaje a través de itinerarios es un hecho familiar dentro de la ciencia geográfica que cuenta con numerosas publicaciones (Mínguez, 2011; Crespo, 2012; Alcolea y otros, 2014).

La plataforma de AGOL y sus crecientes posibilidades de integrar imágenes (fotografías y vídeos), gráficos, datos y comentarios sobre la cartografía, han permitido organizar varios itinerarios geográficos por España invitando a la reflexión sobre una gran diversidad de paisajes. Una herramienta ideal para conocerlos y valorarlos, paso previo imprescindible a su conservación efectiva, es el itinerario (Doctor, 2011). Con ello se consigue reforzar el pensamiento espacial, el respeto y valoración del medio ambiente y el patrimonio, las competencias tecnológicas y espaciales y la alfabetización científica en el marco de la innovación docente y del aprendizaje durante toda la vida (Lázaro, Alcolea y Palacios, 2013).

Lo que aquí se expone son los resultados del Proyecto de Innovación y Mejora de la Calidad Docente de la UCM «Aprender Geografía de España con la Web 2.0» (PIMCD 165/2013) coordinado por el profesor Alcolea. En este proyecto se planteó la creación de materiales propios para una mejor aproximación al paisaje a través de itinerarios geográficos con una duración máxima del recorrido a pie de cuatro horas. Con ellos, empleando tecnología ESRI y la técnica del storytelling, se aborda una mejor comprensión de ese territorio y la profundización en algunos de los conceptos principales de la ciencia geográfica.

Se ha realizado un trabajo colaborativo, con responsabilidades personales y un consenso en los aspectos relevantes a trabajar en el mismo. La responsabilidad de cada itinerario ha sido llevada a cabo por un experto en la localidad, como se señala más adelante, si bien la 
parte técnica en AGOL, ha sido coordinada y realizada por la profesora Lázaro, administradora de la plataforma para organizaciones de ArcGIS Online (ESRI) de la Universidad Complutense de Madrid, aunque se podría haber hecho también en la versión gratuita de la plataforma, ya que no se han creado servicios a partir de los geodatos aportados. La creación de servicios es una de las más importantes diferencias entre la versión gratuita y la versión de pago de la plataforma. El no haberlo hecho así tiene la desventaja de que no es posible actualizar la base de datos y, por tanto, sólo es posible actualizar los datos de las capas existentes añadiendo o creando nuevas capas de datos.

En todos los itinerarios el título está formado por el nombre de la localidad y el aspecto más relevante trabajado en ella, que sería el tema del storytelling reflejado en la cartografía. A continuación se citan los itinerarios realizados en este proyecto ordenados según la provincia a la que pertenecen. Entre paréntesis se cita al autor ${ }^{4}$, responsable de los contenidos de cada uno de ellos:

Madrid:

- Madrid, recinto histórico y murallas medievales (Dr. D. Miguel Ángel Alcolea Moratilla)

- Getafe, las funciones urbanas (Dra. Dña. María Luisa Gómez Ruíz)

- Alcalá de Henares, Ciudad Patrimonio de la Humanidad (Dra. Dña. María Eulalia Ruiz Palomeque)

- Laguna de Peñalara, paisaje natural de alta montaña (D. Luis Alfonso Cruz Naïmi)

Otras provincias:

Ávila:

- Ávila, tradición, historia y retos actuales (Dra. Dña. María Teresa Palacios Estremera)

Badajoz:

- Badajoz, ciudad de frontera (D. Isaac Buzo Sánchez)

Granada:

- Granada, la ciudad y sus jardines (D. Miguel González Castañe)

Jaén:

- Baños de la Encina, un patrimonio a conservar (Dra. Dña. María Luisa de Lázaro y Torres)

León:

- León, las plazas del casco histórico (Dra. Dña. María Jesús González González)

Málaga:

- Antequera, religiosidad, simbología y reconquista (Dr. D. José Jesús Delgado Peña)

- Axarquía Oriental, patrimonio histórico y natural del paisaje (D. José Manuel Crespo Castellanos)

Pontevedra:

- Pontevedra, vestigios del pasado, pilares del presente y del futuro (Dña. María Sotelo Pérez)

4 La mayoría de los autores pertenecen al Grupo de Investigación UCM (931335) Innovación didáctica para la enseñanza de la Geografía en el marco del EEES coordinado por la Dra. D ${ }^{a}$ María Luisa de Lázaro y Torres. 


\section{Zaragoza:}

- Zaragoza, los restos romanos de su casco histórico (Dr. D. Rafael De Miguel González)

Todos estos itinerarios pueden ser visualizados en un «carrusel» dentro de AGOL (figura $\mathrm{n}^{\circ}$ 2) con la posibilidad de compartirlos, añadir comentarios, registrar el número de visitas, y añadir otra información en la forma señalada anteriormente.

\section{Aprender Geografía de España con la Web 2.0}

\section{Cloud fieldwork for learning Spanish geography}

Se muestran aquí los itinerarios elaborados para mostrar algunos paisajes de España

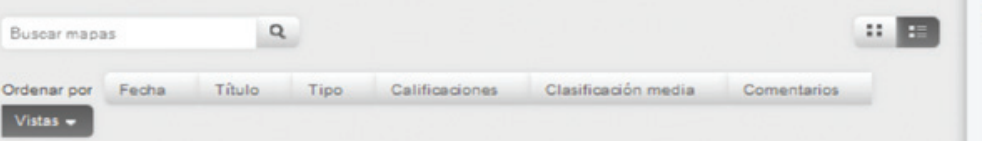

a

\section{Vistas -}

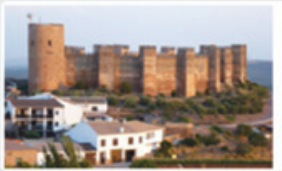

Baños de la Encina, un patrimonio a conservar

Web Mapoing Agolication de milazaro_UCM. Last modified oet 15. p.m.

Se propone un itinerario que muestra los valores paisajisticos y patrimoniales de la

localidad

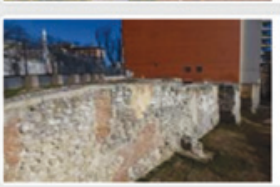

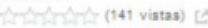

Madrid, el recinto histórieo $y$ las murallas medievales

Web Mapping Appication de milazaro_UCM. Last modified dio 28, a m

Itinerario por donde discumian las murallas medievales de Madrid

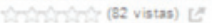

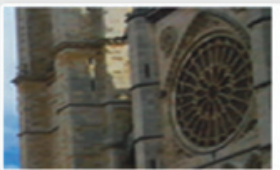

León, las plazas del casco histórico

Web Mapoing Acplication de mponza24_ucmadrid. Last modifed oct 3, per

Se propone un itinerario que muestra las plazas del casco histórico de León

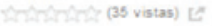

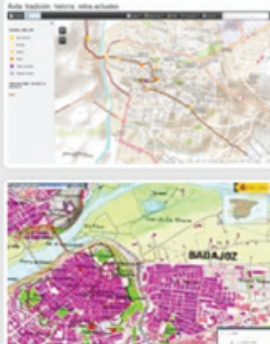

Avila: tradición, historia, retos actuales

Wres Mapging Agplication de mlizaro_UCM. Last modified oet 3. p.m

Itinerario por ta cludad de Ávila

thimintally (30 vistas) $E Z$

Badajoz, ciudad de frontera

Web Mapping Application de buzo_ucmadid. Last modified sep 28, p m

Se propone un itinerario que muestra los valores patrimoniales de la localidad

(22 vistas) $E^{\prime}$

Fuente: Elaborado por M.L. Lázaro a partir de los datos de los itinerarios aportados en el proyecto PIMCD 165/2013. Disponible en: http://ucmadrid.maps.arcgis.com/apps/PublicGallery/index.html?appid=e855e451f33d4d8d97b71ac 22a0b7521\&group=8a3cfca16dc74e70aebdb0e0a3d0cf39 
Para la elaboración de los itinerarios ha sido necesario un trabajo previo sobre distintos aspectos relacionados con la referencia espacial o geolocalización, lo que se ha realizado a partir de los dispositivos móviles (cámaras de fotos con GPS, aplicaciones GPS de los teléfonos inteligentes y otros dispositivos GPS). Con ellos se ha obtenido en el trabajo de campo el raw track o itinerario para cada uno de ellos en formato GPX. Estos primeros itinerarios tomados sobre el territorio han sido redibujados en el visor Iberpix del Instituto Geográfico Nacional para mejorar su presentación e integrarlos posteriormente en la plataforma de AGOL.

La metodología de trabajo se ha basado en la selección de imágenes representativas de cada uno de los itinerarios y de su comentario siguiendo unas pautas comunes que ayuden a una mejor comprensión del paisaje que representan, estas son (Alcolea y otros, 2014): una introducción general en donde se explican los objetivos y la justificación de la elección del paisaje a abordar mediante las paradas del itinerario, imagen representativa de la parada y referencia espacial de la misma, descripción, propuesta didáctica o cuestiones a resolver por los estudiantes y algunos enlaces a páginas web que ayuden a una mejor comprensión del paisaje. El fichero resultante con todos los datos ha sido guardado en formato csv (delimitado por comas) y con codificación UTF-8, para evitar problemas con los signos de puntuación.

El punto exacto desde el que se ha tomado cada una de las imágenes que constituyen las paradas principales del itinerario, se ha obtenido en AGOL directamente para evitar problemas con los cambios de proyección; por tanto, el datum y la proyección empleada han sido los propios de la plataforma, WGS84 Web Mercator (Auxiliary sphere) y el formato de las coordenadas geográficas ha sido en grados y décimas de grados.

Con estos itinerarios se pretende ayudar a entender los paisajes representados en ellos, trabajando la observación, el análisis, el comentario y la valoración de estos paisajes, su sostenibilidad y su realidad patrimonial. Para ello se plantean algunas otras cuestiones que invitan a la reflexión sobre los mismos de la que se deriven posibles pautas de actuación (Kerski, 2011). Toda esta información se ha integrado en las ventanas emergentes que el programa permite crear.

Aunque los itinerarios han sido diseñados y establecidos por cada uno de los expertos, se experimentó con la posibilidad que ofrecía la plataforma de AGOL de construcción automática de itinerarios a partir de una serie de paradas o puntos dados elegidos por los expertos por su especial interés. Los itinerarios así realizados han supuesto una aproximación a cada uno de los itinerarios definitivos, si bien con algunos inconvenientes que pasamos a enumerar. La capa de redes ofrecida no se adecuaba completamente a los objetivos del trabajo, ya que ha sido necesario en algunos casos establecer paradas en calles que están formadas por escaleras, calles sin salida y otros lugares que no contaban con posibilidad de ser incluidos en la ruta de forma automática y que son bastante comunes en muchos de los cascos históricos de nuestras ciudades. Por otro lado, las rutas establecidas en paisajes rurales o forestales, con frecuencia han sido diseñadas por senderos no transitados por vehículos.

Se muestra un ejemplo de la comparativa entre el trazado automático del itinerario realizado para la ciudad de Ávila (figura 3a) y el itinerario definitivamente tomado con un dispositivo GPS (figura 3b). 
Figura 3

ITINERARIO DE ÁVILA
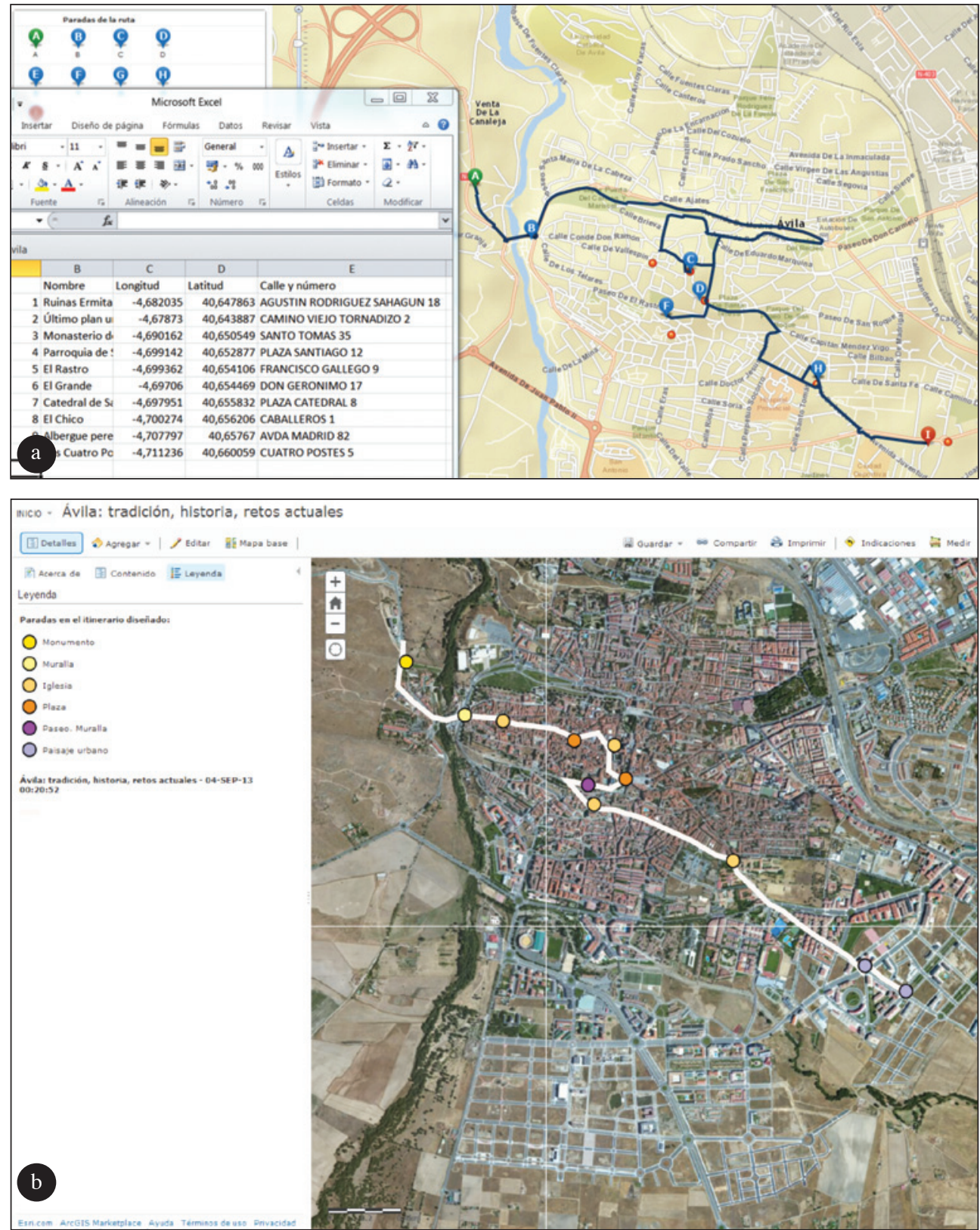

Fuente: Elaborado por M.L. Lázaro en ArcGIS Online a partir de los datos aportados por M.T. Palacios. El mapa de la figura 3.b está disponible en: http://ucmadrid.maps.arcgis.com/apps/OnePane/basicviewer/index.html?appid=63 20f9efcc2d462cb5547a74d5a85ac8 
Además de las imágenes y los demás recursos que la acompañan, es posible añadir otra información en las rutas, como el perfil topográfico de las mismas, desde el que se puede deducir si existe alguna dificultad en su recorrido derivada de una mayor inclinación de la superficie del terreno. También es posible asociar otros elementos que existan en la Web y resulte de interés enlazarlos con el itinerario establecido para una mejor comprensión de los cambios más recientes en el paisaje que representa, como por ejemplo la opción de poder visualizar imágenes de años anteriores, como las imágenes aéreas del vuelo americano de 1956 o de otras ortoimágenes digitales posteriores, gracias a las conexiones OGC especialmente desarrolladas en relación a la visualización o WMS (Web Map Service), que permite observar situaciones anteriores de esos paisajes. Entre otras cosas, se puede observar el crecimiento de las superficies artificiales en general y del poblamiento en particular, a partir de activar o desactivar las capas derivadas de las conexiones citadas o de visualizarlas en ventanas contiguas dentro de la misma pantalla, tomando otra plantilla de la plataforma de ESRI.

Esta metodología de trabajo integrando el comentario de imágenes y la tecnología, es especialmente útil para presentar los resultados de los trabajos de investigación en conferencias, jornadas o en la propia aula docente de la universidad, como se ha hecho en los casos que aquí se presentan. Las prácticas externas que se realizan en la Universidad pueden contar así con una herramienta SIG de gran utilidad, que puede estar unida a las crecientes posibilidades de trabajo colaborativo en la Nube que ofrece Google Drive, el campus virtual... favoreciendo además del trabajo colaborativo, el aprendizaje por proyectos en nuestras aulas.

La gestión y agrupación de los geodatos en forma de itinerarios geográficos y culturales unidos a los SIG en la Nube añaden nuevos resultados a una investigación, por su novedosa forma de visualización de los datos y las posibilidades que ofrece la navegación por ellos, que en el caso de los itinerarios establecidos amplía las formas de aproximación al paisaje, para su conocimiento, valoración y actuación en el territorio. Además, esta forma de elaborar itinerarios supone una constante adaptación a las innovaciones, que es un elemento esencial en el aprendizaje durante toda la vida, y permite una mayor interacción entre los investigadores y dentro de los procesos de enseñanza-aprendizaje, por lo que lo consideramos extrapolable a otras realidades espaciales y ciudadanas, profesionales y educativas.

\section{LOS PAISAJES AGRARIOS DE ESPAÑA A TRAVÉS DE LA ELABORACIÓN EN LA NUBE DE UNA CARTOGRAFÍA COLABORATIVA}

En este segundo caso, y dado que la investigación reciente en Geografía ha mostrado un creciente interés en las formas en que se lleva a cabo la ruralidad, se ha trabajado con los paisajes agrarios que han experimentado un proceso de transformación y modernización con elementos como calidad, innovación, biodiversidad, paisaje, patrimonio... (Foronda, 2013). Cada vez más la investigación muestra un creciente interés por los paisajes rurales, integrando actividades de todos los sectores de la actividad económica, si bien son los paisajes agrarios la razón de ser y el origen de la mayoría de los paisajes rurales. Por otro lado existen distintas aproximaciones o miradas sobre los paisajes agrarios (Delgado y Ojeda, 2009), lo que amplía y diversifica su estudio. El conocimiento del paisaje a través de la descripción y comentario de una imagen es una línea de investigación de gran interés (Palacios y Lázaro, 2013) que potencia la técnica del storytelling. 
La experiencia ha sido realizada con los alumnos de la asignatura de Geografía en el Máster Universitario en Formación del Profesorado de Educación Secundaria Obligatoria y Bachillerato, especialidad de Geografía e Historia, curso académico 2013-2014, con la finalidad de que los futuros profesores lleguen al aula escolar sabiendo qué posibilidades ofrece la Nube en el conocimiento territorial y en la adquisición de competencias docentes, espaciales y digitales. Para ello nos centramos especialmente sobre cómo:

- Acceder a los geodatos de los diversos organismos oficiales, como por ejemplo el Instituto Geográfico Nacional y el Ministerio de Agricultura, Alimentación y Medio Ambiente, entre otros.

- Crear los propios geodatos para su empleo integrados con otros datos, lo que implica un mejor conocimiento de las herramientas de geolocalización.

- Dotar al alumno, futuro profesor, de herramientas que permitan una mejor comprensión del territorio a partir de su visualización y navegación para que tengan una repercusión posterior en el aula escolar.

- Conocer el mundo agrario a partir de la narración (storytelling) de las vivencias de los estudiantes sobre esos paisajes creando un mapa-resumen colaborativo con la información aportada por ellos, con cuestiones concretas para el trabajo en el aula universitaria y no universitaria.

La metodología empleada ha implicado trabajar en la Nube, lo que se deriva del avance tecnológico en las universidades y del emergente concepto de smart learning. Cada alumno debía elegir una imagen seleccionada de forma cuidada y realizada por él mismo o tomada de la Red a través de una URL. No debía repetirse ni la imagen, ni el municipio en el que se ubicara, lo que aseguraba una mayor variedad de paisajes. Cuando el estudiante no tenía los derechos de autor sobre la imagen, porque no la había hecho él mismo, ha tenido que investigar la forma de citar, lo que ha sido un aprendizaje adicional en el proyecto realizado. De hecho fue necesario aportar orientaciones sobre diversos aspectos, como por ejemplo sobre las licencias Creative Commons, que muchos desconocían e incluso aportar un listado de direcciones en la Nube para facilitar la localización de imágenes susceptibles de reutilizar en su trabajo respetando los derechos de la propiedad intelectual.

La información sobre la imagen elegida se debía reflejar primero en una ficha de trabajo en un procesador de texto convencional, que revisó previamente el profesorado de la asignatura. Esta ficha debía contener la imagen y sus coordenadas geográficas tomadas de AGOL, un título significativo para el paisaje agrario y sus actividades principales, de forma que posteriormente fuera posible organizar los paisajes seleccionados; las características generales y las unidades de paisaje existentes en ella (se aportaron previamente los principales conceptos sobre el paisaje agrario); unas cuestiones o preguntas significativas sobre ese paisaje que posibilitara ampliar la reflexión sobre ese territorio y un enlace a alguna página de Internet para ampliar la información aportada.

Una vez revisada la información ésta se integraría en la hoja de cálculo de Google Drive, abierta por el profesor en la Nube y compartida por todos los estudiantes a través de un enlace. Esta herramienta está accesible a través del correo electrónico institucional de la UCM. Las posibilidades del trabajo colaborativo se amplían con esta tecnología al permitir que todos los 
estudiantes trabajen en un mismo documento. Para la correcta publicación del mapa en la Nube es imprescindible que en esta hoja de cálculo se recoja la localización de la imagen comentada mediante las coordenadas geográficas en décimas de grado tomadas de ArcGIS Online, para evitar los problemas derivados del manejo del datum, como hemos señalado en varias ocasiones. La URL de la imagen seleccionada, si ésta fue tomada de una licencia Creative Commons de Internet, o la URL del servidor en el que estuviera depositada la propia imagen original del alumno, que en este caso se subieron previamente al Campus Virtual de la UCM y los créditos o autor de la misma, siguiendo una política respetuosa con los derechos de autor.

En resumen, se recogen los datos de la ficha realizada por los estudiantes en un formato que pueda ser leído sin problemas en AGOL y se pueda integrar a la plataforma sin dificultades, lo que es posible mediante la exportación de la hoja de cálculo de Google Drive directamente a formato csv (delimitado por comas) y, como en el caso de los itinerarios, con codificación UTF-8, para evitar problemas con los signos de puntuación. A partir de esta hoja se realizaría el mapa colaborativo que daría lugar a las exposiciones y el debate en el aula, como se explica posteriormente.

Los datos así recogidos y visualizados en un mapa (figura 4) ofrecen una panorámica de la situación agraria española a través de los conocimientos aportados por los alumnos. Esto supone no sólo unos contenidos importantes, en ocasiones de primera mano, de la situación cotidiana existente en determinadas localidades, sino del área geográfica más conocida por los estudiantes.

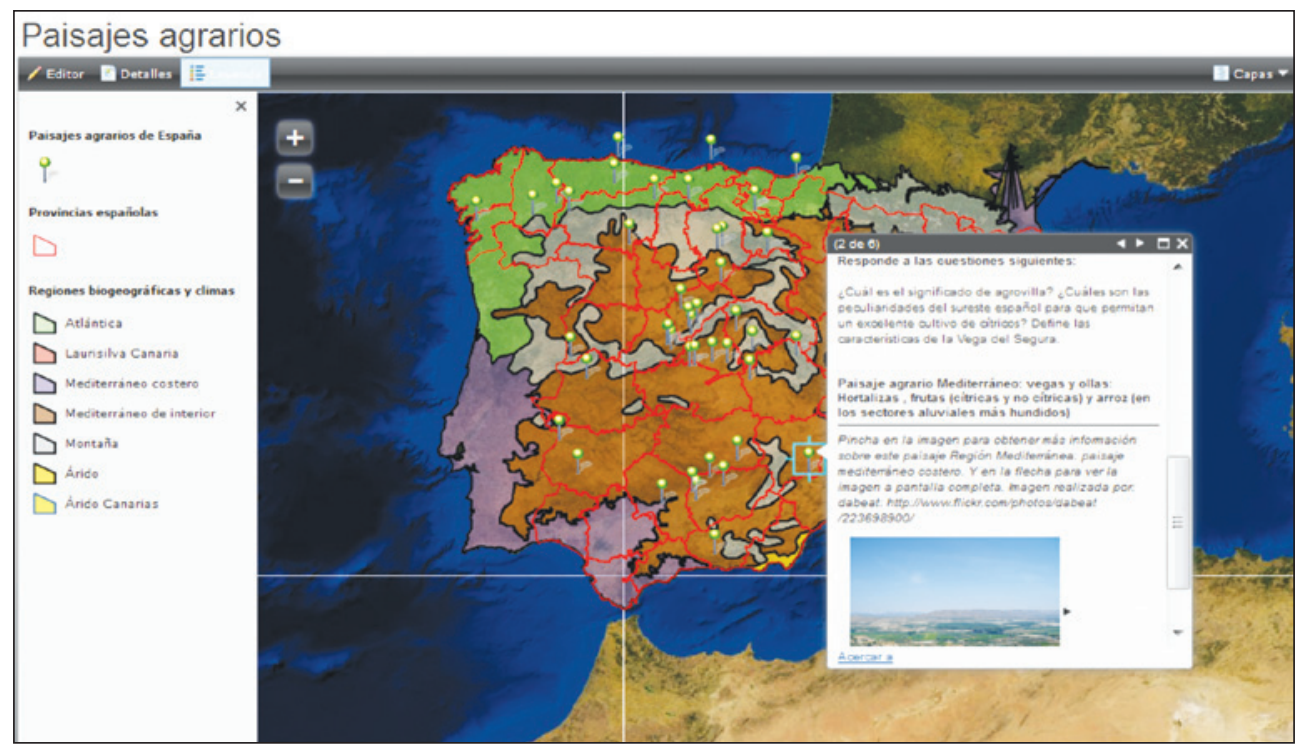

Fuente: Elaborado por M.L. Lázaro en ArcGIS Online a partir de los datos aportados por los estudiantes de Geografía del Máster Universitario en Formación del Profesorado de Educación Secundaria Obligatoria y Bachillerato (curso 2013-2014). http://ucmadrid.maps.arcgis.com/apps/OnePane/basicviewer/index .html?appid=db455dc4e421 4f58a491b8858b9e0af5 
Se detectaron algunas lagunas que pueden ser subsanadas por otros medios: la explicación del profesor o la invitación a que otros profesores de esas realidades territoriales realicen el mismo trabajo con sus propios alumnos y enriquecer así la cartografía creada. De este modo, surge el proyecto de Innovación y Mejora de la Calidad de la Docencia (PIMCD 98/2014), que se desarrolla actualmente en la UCM, coordinado por la Doctora Lázaro. La facilidad, que la herramienta ofrece, de poder trabajar en la misma cartografía desde distintos emplazamientos potencia el trabajo cooperativo y reafirma las ventajas del trabajo en la Nube. Supone un trabajo desde cualquier lugar (ubicuidad) y desde cualquier dispositivo, en cualquier momento, sin grandes requisitos de hardware... Estos mapas permiten así recoger no sólo los resultados del trabajo en el aula, sino también los resultados de una investigación. El fácil manejo de la cartografía con la opción de poder cambiar la categorización de los resultados de forma ágil y visualizar los datos atendiendo a distintos criterios, así como navegar por la cartografía sin la necesidad de un elevado número de mapas convencionales en papel, permite obtener nuevos resultados y conclusiones en la investigación y en la docencia.

La exposición realizada por cada uno de los alumnos organizada por áreas biogeográficas y su aprovechamiento agrario predominante, así como el debate final sobre el mapa obtenido, terminó por hacer aflorar algunos de los problemas principales del sector agropecuario en España con información de primera mano de algunos de sus protagonistas. La mayoría de los alumnos eligieron un área muy bien conocida por ellos mismos, aplicando los contenidos científicos pertinentes.

De esta forma el trabajo cooperativo y colaborativo realizado se convirtió en un aprendizaje vivencial con la participación de todos y cada uno de los estudiantes, impulsando el debate. El valor añadido de integrar otras divisiones territoriales relacionadas con el mundo agropecuario, por ejemplo las comarcas agrarias o las comarcas ganaderas, abre nuevas posibilidades, lo que podría ratificar o cuestionar las delimitaciones actualmente existentes. Si bien, tenemos que decir que a pesar de las continuas mejoras de la plataforma, existen todavía algunas limitaciones, como el tener que subir por partes las capas anteriormente citadas si se emplean para toda España, por su excesivo peso, por ejemplo, por Comunidades Autónomas u otro tipo de división necesaria para que sea viable la visualización de esos datos en el mapa.

Con esto se ha pretendido aproximar al alumnado a la visualización de la información geográfica, a saber orientarse y buscar las coordenadas geográficas de una imagen, a profundizar en las relaciones espaciales de los fenómenos y su distribución territorial y a experimentar las posibilidades de un trabajo colaborativo sin precedentes que años atrás hubiera sido impensable realizar por la inexistencia de herramientas que están hoy al alcance de investigadores y profesores. La gran interacción posible y el intercambio de información permitieron, además, conocer la percepción existente sobre el territorio de los propios estudiantes, no sólo para una mejor comprensión del paisaje y las posibilidades de actuación positiva sobre el mismo, sino para observar las carencias en el conocimiento territorial de los propios estudiantes y a partir de ellas construir el conocimiento mediante un aprendizaje significativo.

En cuanto a los resultados, apuntar que los estudiantes adquirieron competencias docentes, espaciales y digitales:

a) Docentes: se ha mostrado una forma novedosa de observar en un solo mapa la percepción que de los paisajes agrarios tenían los estudiantes, trabajando parte de los 
contenidos fuera del aula y ayudando a aproximarse a la tecnología en la propia clase para poder resolver dudas y que en ningún caso la tecnología resultara una barrera. Parte de este trabajo es lo que hoy se conoce como flipped classroom o clase inversa, en la que la clase magistral del profesor se sustituye por el trabajo del alumno fuera del aula, en este caso los contenidos y las fichas realizadas, mientras que en clase se aprendió a utilizar Google Drive, a realizar mapas en AGOL, a exponer su trabajo y a escuchar las exposiciones de los demás fomentando así un debate que ha permitido un aprendizaje vivencial.

b) Espaciales: los estudiantes comprendieron mejor la dinámica territorial de áreas ajenas al lugar en el que viven o al lugar del que provienen a través de las explicaciones de los compañeros, la visualización de las imágenes y el debate posterior.

c) Digitales: los alumnos lograron mejorar su alfabetización digital con fines docentes y aprendieron a hacer mapas que han quedado en la Nube en la plataforma de AGOL disponibles para su empleo y consulta posterior. También aprendieron a buscar datos y a investigar respetando los derechos de la propiedad intelectual para los docentes en Internet.

Otros hechos que demuestran el avance en las competencias adquiridas:

- El modelo ha sido llevado a cabo después por varios alumnos en sus Trabajos de Fin de Máster del Master Universitario en Formación del Profesorado de Educación Secundaria Obligatoria y Bachillerato, especialidad de Geografía e Historia del curso académico 2013-2014 y están en trámite de presentarlos a varios congresos nacionales e internacionales, hecho que no sólo nos ha llenado de satisfacción, sino que muestra el éxito de la experiencia realizada en el aula.

- El examen final que realizaron tuvo resultados muy satisfactorios.

Las ventajas esperadas y demostradas posteriormente han hecho que no se considerara oportuno emplear un grupo de control, que exigía el privar a un grupo de estudiantes del avance y adquisición de las competencias anteriormente citadas.

No podemos decir que la experiencia estuviera exenta de dificultades, ya que la actualización de los servidores empleados necesita revisiones y algunos días no funcionaron, los niveles de partida en la alfabetización digital de los estudiantes eran muy dispares y conseguir que las herramientas informáticas no resultaran una barrera para nadie resultó un gran reto, dado el escaso número de horas lectivas con el que se contaba.

La mayoría de los estudiantes estuvieron entusiasmados con la experiencia, y todos ellos ofrecieron traer sus propios ordenadores portátiles y compartirlos con aquellos alumnos que carecían de ellos, para poder llevar a cabo la experiencia.

\section{VALORACIÓN DE LOS RESULTADOS Y CONCLUSIONES}

Resulta indiscutible la utilidad de los SIG para mejorar los resultados del aprendizaje con datos que tengan un componente geográfico. Además, la creciente integración de los SIG en la Nube añade otras muchas posibilidades tanto para el aprendizaje y como para la investigación, tal y como se ha demostrado a lo largo de la exposición. 
Con este trabajo se ha pretendido aprovechar la oportunidad que algunas tecnologías emergentes están haciendo de la Geografía una ciencia en alza en un mundo que demanda cada vez más herramientas de geolocalización y geoservicios. Con ello se refuerza el pensamiento espacial, las competencias tecnológicas y espaciales y la alfabetización científica.

Podemos concluir con Antonio Moreno (2013) que las transformaciones del proceso de generación de conocimiento geográfico basado en geotecnologías son de tal calado que poseen rango epistemológico, es decir, que implican un entendimiento y una praxis indagatoria nueva y esencialmente distinta de otras que existen y han existido en Geografía, no sólo en investigación, sino que además exigen nuevas metodologías docentes, como se ha pretendido aportar en este trabajo. Algunos autores hablan desde hace algunos años de un nuevo paradigma en geografía (Koutsopoulos, 2008 y 2011).

Todo ello hace que el trabajo realizado tenga un gran potencial, favorecido por las futuras actualizaciones de la propia herramienta utilizada y por su facilidad de empleo en el trabajo colaborativo en las aulas y en la rápida integración de otras capas de datos, imágenes, vídeos, textos, etc.

AGOL facilita así un producto abierto al que, como ya hemos dicho reiteradamente, se pueden añadir nuevos datos (geodatos). Queda demostrada la clara visualización de los datos (geodatos) que ofrece y su facilidad de empleo.

La plataforma ArcGIS Online y la técnica del storytelling, aplicada en nuestro caso a la Nube de Internet, resultan de gran validez para aproximarnos a la realidad territorial y al paisaje a través de itinerarios didácticos y de la cartografía colaborativa, empleando el comentario de imágenes sobre paisajes agrarios.

El potencial del trabajo colaborativo que aquí se ha presentado nos permite ratificar las afirmaciones del blog de A. Fidalgo:

- Compartir el conocimiento contribuye a mejorarlo.

- Usar el conocimiento contribuye a que se genere más conocimiento.

- El conocimiento no se agota al darlo.

- Colaborar en su creación lo perfecciona y todos los que colaboran se lo llevan.

Se aportan de esta manera herramientas que sin duda construyen nuevo conocimiento y ayudarán a los retos profesionales futuros de los estudiantes, profesores y demás profesionales de la Geografía y otras ciencias colaborando con ello con el objetivo OE3 - Objetivo Educación del campus de excelencia de la Universidad Complutense de Madrid.

\section{AGRADECIMIENTOS}

A la aportación de los geodatos resultado de la investigación en innovación educativa de los PIMCD de la UCM en los que han participado el equipo de expertos de los distintos paisajes trabajados, sin cuya aportación hubiera sido imposible avanzar en la experimentación de tecnologías emergentes, al Campus de Excelencia de la UCM, a la red de centros de excelencia digital-earth .eu por el apoyo en innovadoras herramientas SIG, al proyecto School on Cloud (SoC) por la oportunidad de seguir profundizando en estas herramientas y a la buena 
acogida del proyecto por parte de los estudiantes de Geografía del Máster Universitario en Formación del Profesorado de Educación Secundaria Obligatoria y Bachillerato de la UCM del curso 2013-2014.

\section{BIBLIOGRAFÍA}

ALCOLEA, M.A. y otros (2014): «Aprender Geografía de España con la Web 2.0» (PIMCD 165/2013), en Proyectos de Innovación y Mejora de la Calidad Docente 2013. Vicerrectorado de Evaluación de la Calidad. Universidad Complutense de Madrid.

ALTERIO, M. (2002): The Higher Educatio Academy. Using storytelling to enhance student learning. Accesible el 3 de Febrero de 2014 en http://www.heacademy.ac.uk/assets/documents/resources/database/id471_using_storytelling_to_enhance_learning.pdf

BEDDES, R. y JOHNSON C. (Eds.) (1988): Only One Earth: A Multimedia Education Pack, WWF-UK, Godalming, Surrey.

CAMPANO, L.F. (Dir.) (2009): Atlas de los Paisajes de la Región de Murcia. Consejería de Obras Públicas y Ordenación del Territorio. Accesible el 31 de Julio de 2014 en http:// www.sitmurcia.es/paisaje/publica/atlasmur/AtlasPaisajeRegionMurcia.pdf

CRESPO, J.M. (2012): «Un itinerario didáctico para la interpretación de los elementos físicos de los paisajes de la Sierra de Guadarrama», Didáctica Geográfica n ${ }^{\circ} 13,15-34$.

DELGADO, B. y OJEDA, J.F. (2009): «La comprensión de los paisajes agrarios españoles. Aproximación a través de sus representaciones», Boletín de la Asociación de Geógrafos Españoles, $\mathrm{n}^{\circ}$ 51, 93-126.

DE MIGUEL, R. (2013): «Aprendizaje por Descubrimiento, Enseñanza Activa y Geoinformación: Hacia una Didáctica de la Geografía Innovadora». Didáctica Geográfica, nº 14, 17-36.

DE MIGUEL, R. (2014): «Concepciones y usos de las tecnologías de información geográfica en las aulas de ciencias sociales». Iber. Didáctica de las Ciencias Sociales, Geografía e Historia, $\mathrm{n}^{\circ} 76,60-71$.

DOCTOR,A.M. (2011): «El itinerario como herramienta para la puesta en valor turístico del patrimonio territorial», Cuadernos de Turismo, $\mathrm{n}^{\circ} 27,273-289$.

ECK, J. (2006): An analysis of the effectiveness of storytelling with adult learners in supervisor management. University of Wisconsin-Stout. Accesible el 3 de Febrero de 2014 en http://www2.uwstout.edu/content/lib/thesis/2006/2006eckj.pdf

EGAN, K. (1993): Narrative and learning: A voyage of implicatons. Linguistics and Education, $5,119-126$.

ESPEJO, C. y LÓPEZ DE LOS MOZOS, M.A. (2012): «El paisaje de Madrid en la obra de Antonio López García», Nimbus, nº 29-30, 217-232.

FIDALGO, A. «1 y 1 no es igual a $1+1 »$ Blog: http://innovacioneducativa.wordpress. com/2014/03/07/1-y-1-no-es-igual-a-11/

FORONDA, C. (2013): «La investigación rural del siglo XXI en España», Boletín de la Asociación de Geógrafos Españoles, $\mathrm{n}^{\circ}$ 61, 257-265.

GONZÁLEZ, M.J. y LÁZARO, M.L. (2011): «La geoinformación y su importancia para las tecnologías de la información geográfica».Ar@cne. Revista electrónica de recursos en Internet sobre Geografía y Ciencias Sociales. Barcelona: Universidad de Barcelona, $\mathrm{n}^{\circ}$ 148, 1 de junio de 2011. <http://www.ub.es/geocrit/aracne/aracne-148.htm>. 
HO, Q.; ÅSTRÖM, T. y JERN, M. (2013): «Geovisual analytics framework integrated with storytelling applied to HTML5» comunicación presentada a la conferencia de AGILE celebrada en Lovaina (Bélgica) en mayo de 2013. http://ncva.itn.liu.se/resources/publications/1.523791/GeovisualAnalyticsandStorytellingAppliedtoRegionalStaticticsUsingHTML5-AgileShortpaper.pdf

JUNTA DE ANDALUCÍA (2007): Observatorio virtual del paisaje mediterráneo. Consejería de Obras Públicas y Transportes.

KERSKI, J.J. (2011): «Sleepwalking into the Future - The Case for Spatial Analysis Throughout Education» en Jekel, T, Koller, A., Donert, K. \& Vogler, R. (Eds.) Learning with GI 2011. Herbert Wichmann Verlag, VDE VERLAG GMBH, Berlin/Offenbach, 2-11.

KOUTSOPOULOS, K. (2008): «What's European about European Geography? The Case of Geoinformatics in Europeanization», Journal of Geography in Higher Education, 32 (1), $7-14$.

KOUTSOPOULOS, K. (2011): «Changing paradigms in Geography», European Journal of Geography, 1, $54-75$.

LAMBERT, J. (2013): Digital Storytelling: Capturing Lives, Creating Community. New York. Routledge.

LÁZARO, M.L. de; ALCOLEA, M.A. y PALACIOS, M.T. (2013): «Dispositivos móviles: una herramienta para el aprendizaje en Geografía y en los trabajos de campo» en Ángel Fidalgo Blanco y María Luisa Sein-Echaluce Lacleta (Eds) Actas del II Congreso Internacional sobre Aprendizaje, Innovación y Competitividad. Universidad Politécnica de Madrid, 685-690.

LÁZARO, M.L. de (Coord.) (en prensa) «Aprender Geografía con la Web 2.0 a través de la evolución de los paisajes agrarios de España» (PIMCD 98/2014).

LEE, J. y BEDNARZ, R. (2009) Effect of GIS Learning on Spatial Thinking, Journal of Geography in Higher Education, 33 (2), 183-198. DOI: 10.1080/03098260802276714

LICERAS, Á., (2003): Observar e interpretar el paisaje: estrategias didácticas. Grupo Editorial Universitario.

LIVO, N. y RIETZ, S. (1986): Storytelling: Process and Practice, Libraries Unlimited, Colorado.

MATA, R. y SANZ, C. (Dir.) et al. (2003): Atlas de los Paisajes de España. Ed. Ministerio de Medio Ambiente, Madrid.

MÍNGUEZ, M.C. (2010): «El paisaje como objeto de estudio de la Geografía. Un itinerario didáctico en el marco de la semana de la ciencia de la Comunidad de Madrid». Didáctica Geográfica, no 11, 37-62.

MOLINERO, F.; OJEDA, J. y TORT, J. (2011): Los paisajes agrarios de España. Caracterización, evolución y tipificación. Madrid. Ministerio de Medio Ambiente.

MORENO, A. (2013): «Entendimiento y naturaleza de la cientificidad geotecnológica: una aproximación desde el pragmatismo epistemológico». Investigaciones Geográficas, $\mathrm{n}^{\circ}$ 60, 5-36.

PALACIOS, M.T. y LÁZARO, M.L. de (2013): «El patrimonio natural, artístico y cultural a través del análisis de imágenes» en Moreno, M.C. y otros Retos educativos de la cultura andaluza en una sociedad global, Universidad de Málaga, 25-42. 
PONS, B. (Dir.) (2011): Atlas de los Paisajes de Castilla-La Mancha. Ediciones de la Universidad Castilla-La Mancha.

ROBIN, B. (2006). The Educational Uses of Digital Storytelling. Houston: University of Houston.

ZABEL, M.K. (1991): Storytelling, myths, and folk tales: Strategies for multicultural inclusion. Preventing School Failure, 36 (1): 32-34.

ZÁRATE, A. (2011): «Paisajes culturales urbanos, entre la protección y la destrucción». Boletín de la Asociación de Geógrafos Españoles, n 57, 175-194.

VV.AA. (2000): Convenio Europeo del Paisaje. Florencia. 
